Supporting Information for

\title{
Graphoepitaxy-Directed Assembly of Organic Semiconductor Single Crystals into Trellis Structures
}

Kai Zong, ${ }^{\dagger}$ Kaustubh M. Asawa, ${ }^{\ddagger}$ Abigail Circelli, ${ }^{\dagger}$ Nicholas Sparta, ${ }^{\dagger}$ Chang-Hwan Choi, ${ }^{\ddagger}$ and Stephanie S. Lee ${ }^{\dagger, *}$

tDepartment of Chemical Engineering and Materials Science, Stevens Institute of Technology, Hoboken, NJ 07030

抽epartment of Mechanical Engineering, Stevens Institute of Technology, Hoboken, NJ 07030

*stephanie.lee@stevens.edu 


\section{Experimental Methods}

Nanopillar scaffold fabrication: Si wafers were purchased from Addison Engineering and cleaned in piranha solution (mixture of sulfuric acid and $30 \%$ hydrogen peroxide in $3: 1$ volume ratio) for $10 \mathrm{~min}$, followed by de-ionized water for $5 \mathrm{~min}$. Photoresist NR-7 1500 (Futurexx, Inc) was spin coated onto the wafers for $40 \mathrm{~s}$ at $3000 \mathrm{rpm}$, resulting in a film thickness of $1.5 \mu \mathrm{m}$. The wafers were then baked at $120^{\circ} \mathrm{C}$ for $2 \mathrm{~min}$. A He-Cd laser (50 mW and $30 \mathrm{~cm}$ coherence length at a wavelength of $325 \mathrm{~nm}$ ) was used in Lloyd's mirror setup for holographic lithography..$^{1,2}$ The wafers were then post-baked at $100{ }^{\circ} \mathrm{C}$ for one minute before being placed in an undiluted developer solution of RD6, (Futurexx, Inc) for $12 \mathrm{~s}$. The developed patterns were rinsed with de-ionized water for 3 min and blow-dried with nitrogen gas. Using deep reactive ion etching (DRIE) technique, these surfaces were etched in cryogenic conditions $\left(-100^{\circ} \mathrm{C}\right)$, where the developed photoresist patterns served as etch mask. The photoresist layer was then dissolved using piranha solution, followed by thoroughly rinsing it in deionized water and blow-dried with nitrogen gas. These samples were later exposed to oxygen plasma before using it them for next step. CAUTION: piranha solution is a powerful oxidizer and should be handled with extreme care.

Dip coating of perylene crystals from solution onto nanopillar grids: Nanopillar grids were immersed into solutions comprising $0.0625 \mathrm{wt} \%$ perylene in chloroform at a temperature of $70{ }^{\circ} \mathrm{C}$. A syringe pump was used to withdraw the samples at speeds ranging from $0.4-1.0 \mathrm{~mm} \mathrm{~min}^{-1}$. The solutions were covered with aluminum foil to prevent rapid solvent evaporation during the process. 
X-ray diffraction analysis: 2D x-ray diffraction patterns (XRD) were collected using a Bruker AXS D8 DISCOVER GADDS diffractometer with VANTEC 2000 detector. The diffractometer was operated in reflection mode at $40 \mathrm{kV}$ x $40 \mathrm{~mA}$. Data was collected using an incident $x$-ray wavelength of $\lambda=1.5405 \AA$ at an incident angle of $3^{\circ}$ and for a collection time of $600 \mathrm{~s}$ in air at room temperature.
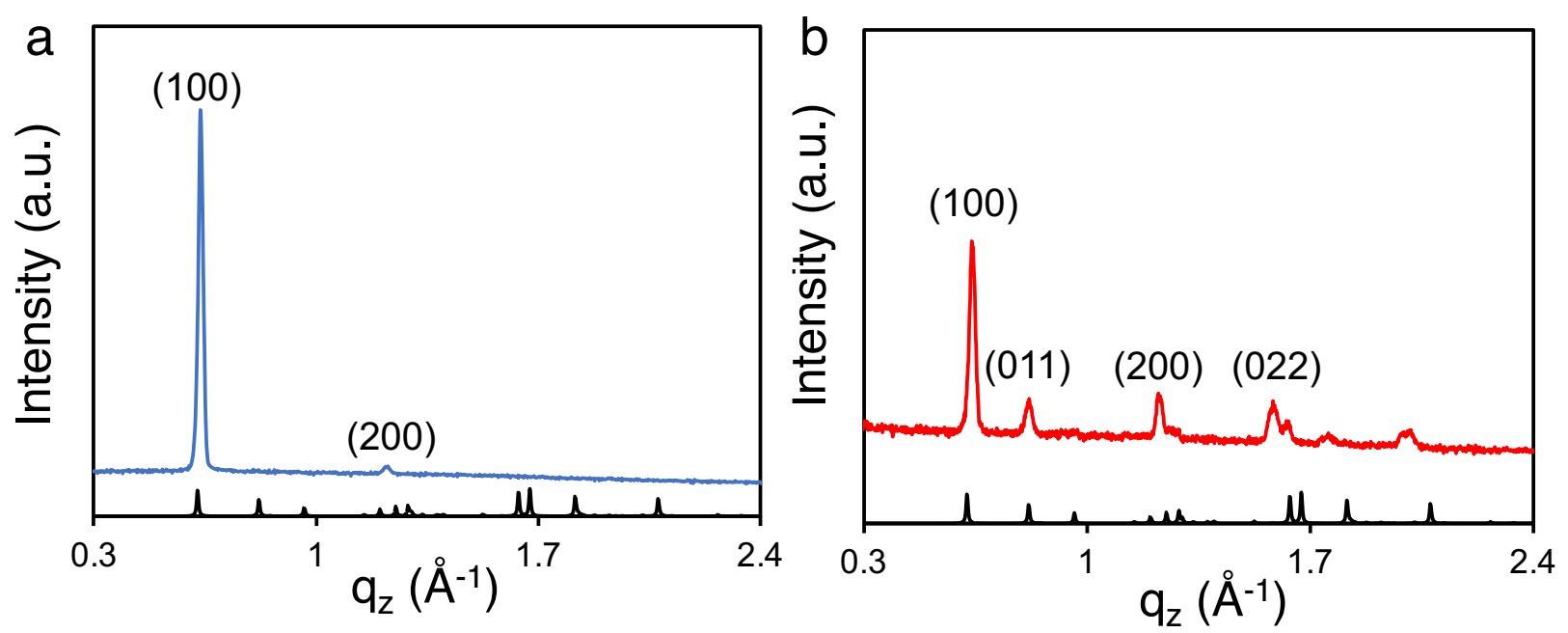

Figure S1. 1-D line traces at $\mathrm{q}_{\mathrm{xy}}=0 \AA^{-1}$ extracted from 2D x-ray diffraction patterns for perylene dip coated onto $\mathbf{a}$, a flat $\mathrm{SiO}_{2}$ substrate and $\mathbf{b}$, a Si nanopillar grid. The simulated powder diffraction pattern of perylene generated from the single crystal structure $^{3}$ is provided for reference. 

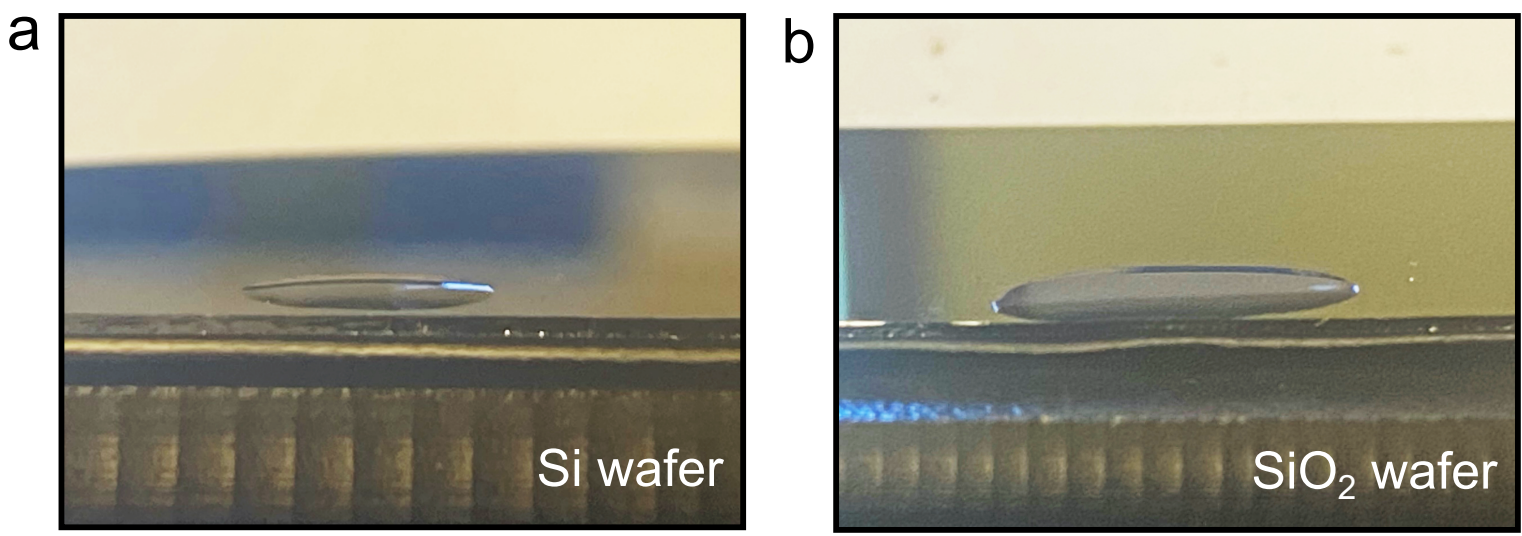

Figure S2. Side profile of a chloroform droplet on both an $\mathrm{Si}$ wafer and $\mathrm{SiO}_{2}$ wafer cleaned by oxygen plasma. Chloroform wets both surfaces, with small observed contact angles.
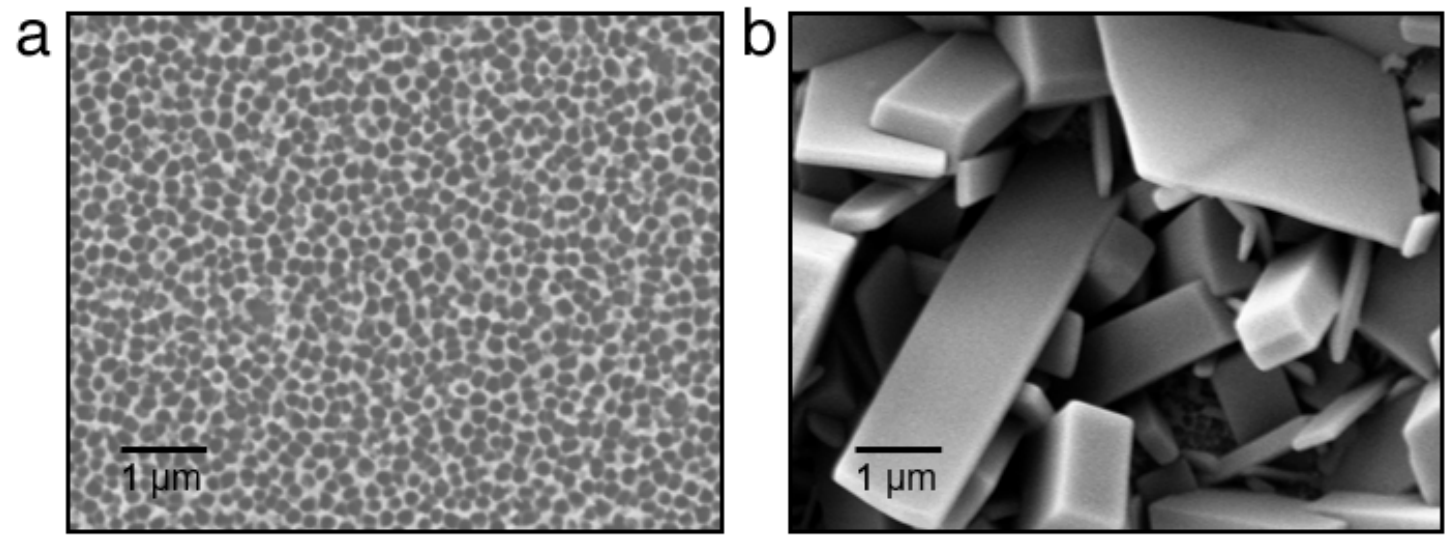

Figure S3. a) Top-view SEM image of an aperiodic anodized aluminum oxide (AAO) scaffold with an average pore dimeter of $100 \mathrm{~nm}$. b) Top-view SEM image of perylene crystals dip coated from a 0.0625 wt\% solution in chloroform onto the AAO scaffold with $S=0.4 \mathrm{~mm} \mathrm{~min}^{-1}$ at a temperature of $70{ }^{\circ} \mathrm{C}$. The crystals display a preferred orientation perpendicular to the substrate but no preferred in-plane orientation. 


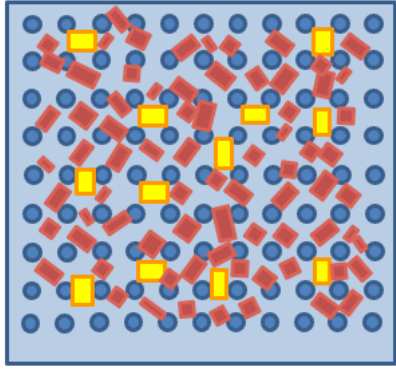

Random nucleation within the grids

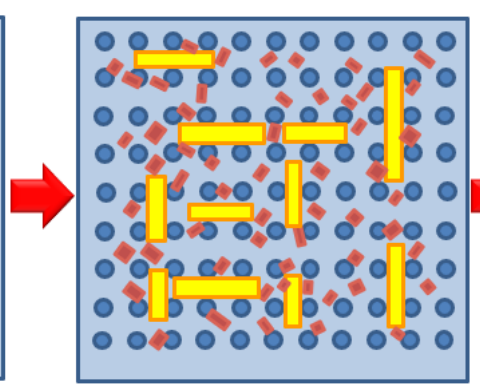

Misoriented crystals dissolve and oriented crystals enlarge

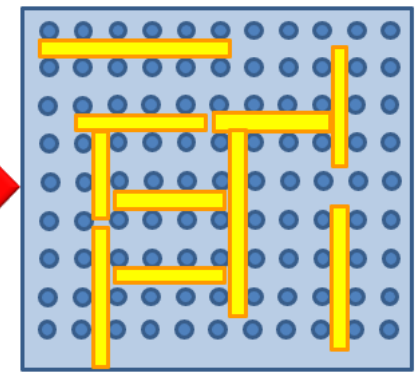

Large, oriented crystals exist in the grids
Misoriented crystals

Oriented crystals

Figure S4. Illustration of the proposed Ostwald ripening process in nanopillar grids as a function of time from a plan-view.

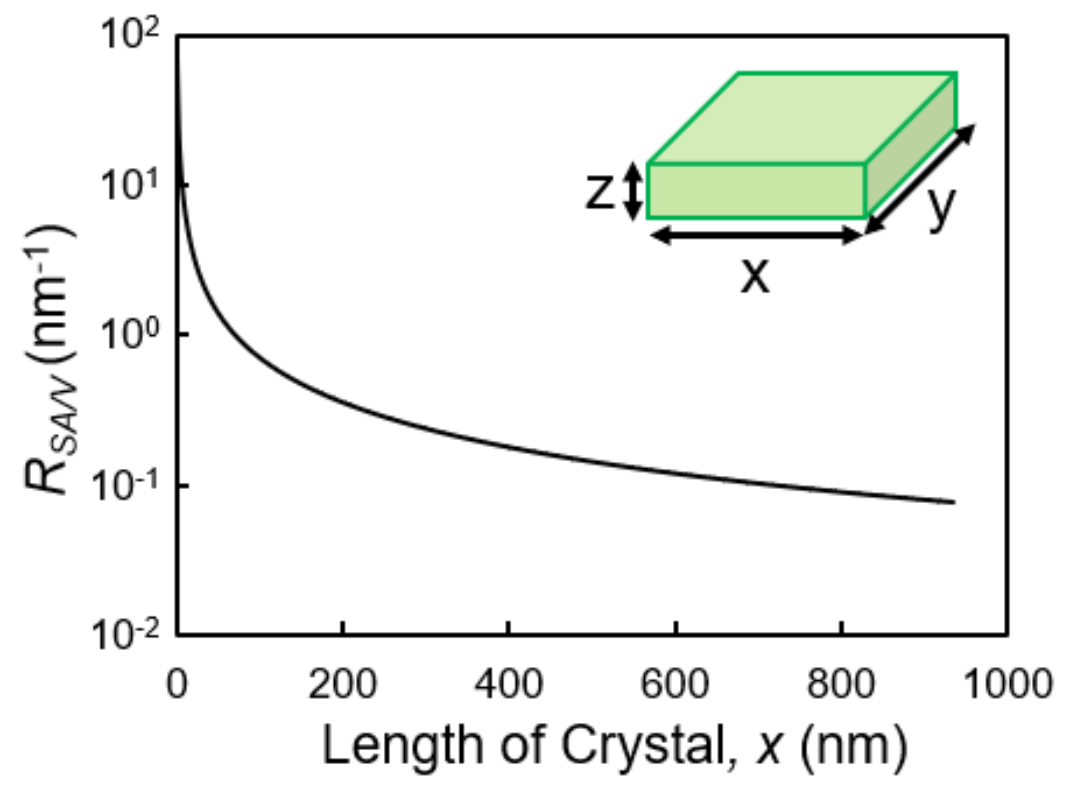

Figure S5. Surface area-to-volume ratio, $R_{S A N}$, for a platelike crystal, as a function of the crystal length, $x$.

Figure S5 displays the surface area-to-volume ratio for a platelike crystal as a function of the length of crystal, $x$. The surface area, $S$, and volume, $V$, were estimated by Equations S1 and S2, respectively: 


$$
\begin{gathered}
S=2 x y+2 y z+2 x z \\
V=x y z
\end{gathered}
$$

Based on experimental evidence, we approximated that $x=y$. We also made the simplification that the height-to-width ratio remains constant as the crystal grows and estimated from scanning electron microscope images that $z / x=0.03$. As observed from the figure, as the crystals grow, $R_{S A N}$ decreases. According to classical nucleation theory, the Gibbs free energy of crystal surfaces is larger than the Gibbs free energy of the crystal volume. As $R_{S A N}$ decreases with increasing crystal size, the overall Gibbs free energy correspondingly decreases. Larger crystals are thus thermodynamically favored compared to smaller crystals. We hypothesize this dependence of the total Gibbs free energy on crystal size drives an Ostwald ripening process.

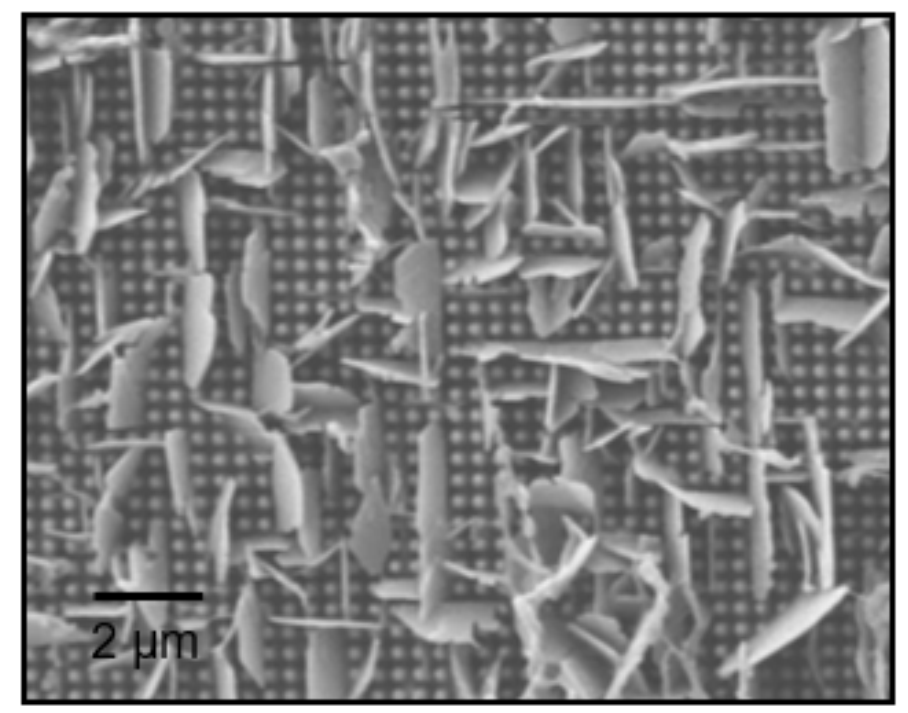

Figure S6. Top-view SEM image of anthracene crystals dip coated from a 0.0625 wt\% solution in toluene onto a Si nanopillar scaffold with $S=0.4 \mathrm{~mm} \mathrm{~min}^{-1}$ at a temperature of $80^{\circ} \mathrm{C}$. 


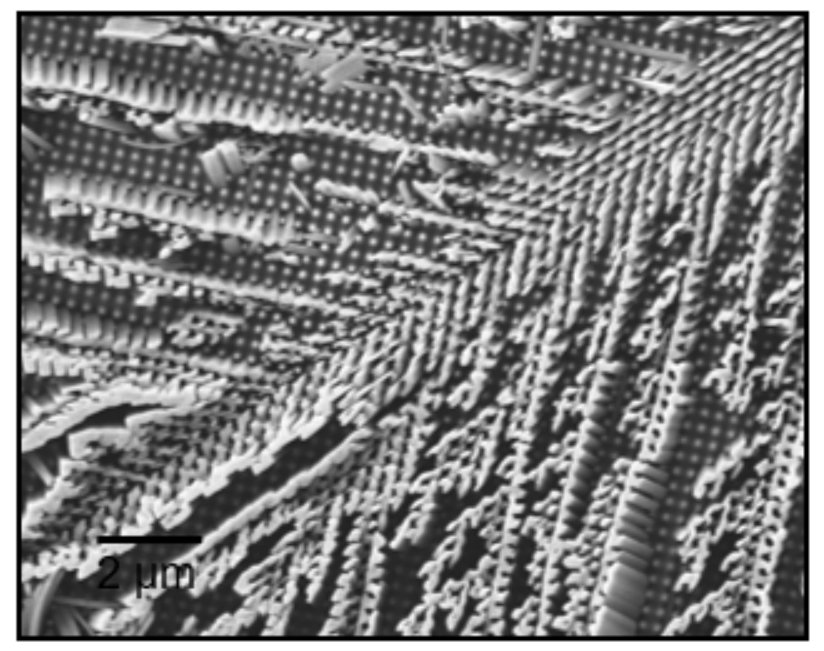

Figure S7. Top-view SEM image of bis(triisopropylsilylethynyl)pyranthrene crystals dip coated from a $0.25 \mathrm{wt} \%$ solution in toluene onto a Si nanopillar scaffold with $S$ $=0.4 \mathrm{~mm} \mathrm{~min}{ }^{-1}$ at a temperature of $80^{\circ} \mathrm{C}$.
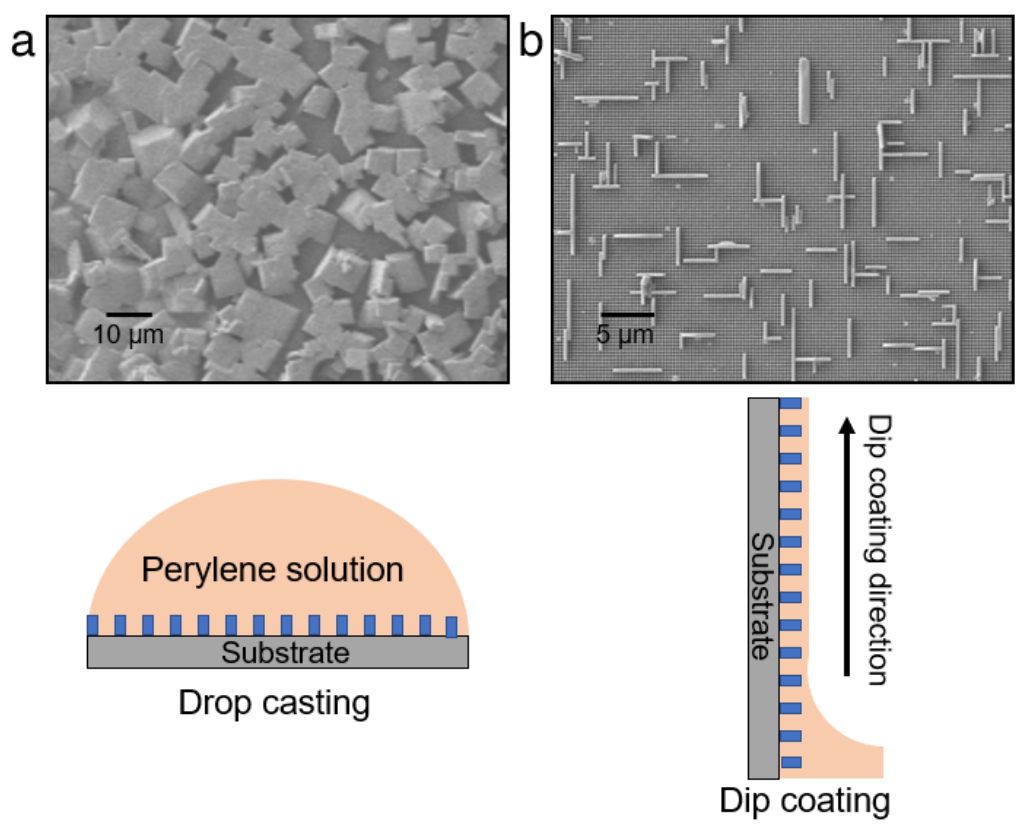

Figure S8. a) Top-view SEM image of perylene crystals drop cast from a 0.0625 wt $\%$ solution in chloroform onto nanopillar grids at a temperature of $70{ }^{\circ} \mathrm{C}$. b) Topview SEM image of perylene crystals dip coated from a $0.0625 \mathrm{wt} \%$ solution in chloroform onto a nanopillar grid with $S=1.0 \mathrm{~mm} \mathrm{~min}^{-1}$ at a temperature of $70{ }^{\circ} \mathrm{C}$. 
Illustrations depict the relative solution heights above the nanopillar grids via the two different deposition methods.

Table S1. Nanopillar scaffold parameters.

\begin{tabular}{|l|c|c|c|}
\hline Parameter & $\mathbf{3 4 0} \mathbf{~ n m ~ g r i d ~}$ & $\mathbf{3 7 0} \mathbf{~ n m ~ g r i d ~}$ & $\mathbf{4 1 0} \mathbf{~ n m ~ g r i d ~}$ \\
\hline Center-to-center spacing & $340 \mathrm{~nm}$ & $370 \mathrm{~nm}$ & $410 \mathrm{~nm}$ \\
\hline Edge-to-edge spacing & $120 \mathrm{~nm}$ & $200 \mathrm{~nm}$ & $300 \mathrm{~nm}$ \\
\hline Height & $650 \mathrm{~nm}$ & $470 \mathrm{~nm}$ & $560 \mathrm{~nm}$ \\
\hline Diameter at top & $220 \mathrm{~nm}$ & $170 \mathrm{~nm}$ & $110 \mathrm{~nm}$ \\
\hline Diameter at bottom & $220 \mathrm{~nm}$ & $280 \mathrm{~nm}$ & $220 \mathrm{~nm}$ \\
\hline
\end{tabular}

\section{References}

(1) Wathuthanthri, I.; Mao, W.; Choi, C.-H. Two Degrees-of-Freedom Lloyd-Mirror Interferometer for Superior Pattern Coverage Area. Opt. Lett. 2011, 36 (9), 15931595.

(2) Mao, W.; Wathuthanthri, I.; Choi, C.-H. Tunable Two-Mirror Interference Lithography System for Wafer-Scale Nanopatterning. Opt. Lett. 2011, 36 (16), 3176-3178.

(3) Perylene | C2OH12 - PubChem

https://pubchem.ncbi.nlm.nih.gov/compound/Perylene. 